The International Journal of Indian Psychology

ISSN 2348-5396 (e) | ISSN: 2349-3429 (p)

Volume 4, Issue 2, No. 89, DIP: 18.01.093/20170402

ISBN: 978-1-365-74162-3

http://www.ijip.in | January-March, 2017

\title{
The Role of Cognitive Factors in Old Age Balance Impairments
}

\author{
Priyanka $\operatorname{Yadav}^{1} *$
}

\section{ABSTRACT}

The ability of human beings to perform more than one task at a time has long been focus of study in the literature on human attention and memory. Older adults are more penalized when they must divide their attention between two input resources i.e input and holding or holding and responding. Falls have potentially devastating physical, social and psychological consequences. Falling is one of the most serious problems associated with ageing. Sensory system deteriorates with age, increased attention is allocated to "HIGHTEN" the signal coming from this system in order to gain necessary information for postural control. Older adults show marked reduction in the ability to perform the postural and cognitive task simultaneously. A study was conducted by physiotherapy and psychology students in a small town of Haryana on institutionalized older adults in which Dual task training under various sets of instruction that is Fixed priority and Variable priority instruction sets effects the balance who are living in residential care facilities. Tinneti Performance Oriented Mobility Assessment (POMA) and Mini mental status examination were used as tools to assess balance and cognitive abilities. Various studies related to the role of cognitive factors in balance impairments were reviewed. Data was analyzed using SPSS 13.0 software packages and paired sample $t$-test within the group and independent sample $t$ - test between the groups with $p-$ value at a significance level of $p<0.05$ was used. The result of the study supported the hypothesis that there is significant improvement in balance of institutionalized elderly people who received dual task training with variable priority instruction set. $(p<0.001)$. The use of validated measurement tool like Tinnetti performance oriented mobility assessment on balance allowed clearer interpretation of the results. There is improvement in TPOMA Scores after 4 weeks training program in both groups. This shows that cognitive factors do play an important role in maintaining balance and coordination. Any impairment related to sensory system or cognition leads to balance impairments and increased risk for falls.

Keywords: Cognitive Factors, Old Age, Impairments

\footnotetext{
${ }^{1}$ Resource Person in Dept. of Psychology, GCW Gurawra, Rewari, Haryana, India

*Responding Author

Received: January 12, 2017; Revision Received: February 11, 2017; Accepted: February 15, 2017

(C) 2017 Yadav P; licensee IJIP. This is an Open Access Research distributed under the terms of the Creative Commons Attribution License (www.creativecommons.org/licenses/by/2.0), which permits unrestricted use, distribution, and reproduction in any Medium, provided the original work is properly cited.
} 


\section{The Role of Cognitive Factors in Old Age Balance Impairments}

The ability of human being to perform more than one task at a time has long been focus of study in the literature on human attention and memory. Older adults are more penalized when they must divide their attention between two input resources i.e input and holding or holding and responding.

Attention is defined as the information processing capacity which is limited for any individual that performing any task requires a given portion of capacity. To act on information, working memory must allocate attention resources. Attention is a finite commodity and any task which require attention compete for this limited capacity, if demand exceeds the capacity performance on one or more task will be diminished.

A strong version of this view predicts that people should have great difficulty doing two tasks at once. A second view is that cognition is limited by the speed with which cognitive system can operate and normal ageing result in slowing of processing. The reduction of attention capabilities due to ageing and competition in motor requirement of maintaining a balance can lead to interference between postural control and mental activity.

Fall defined as an event results when a person coming to rest inadvertently on the ground or other lower levels. Falls have potentially devastating physical , social and psychological consequences .Falling is one of the most serious problems associated with ageing. Nonfatal falls leads to physical injuries like fractures, reduced level of physical activity, loss of confidence and altered life style. Inability to produce an appropriate postural control due to competition for attention resources between the postural system and cognitive task contribute to falls.

Falls involves multiple factors which are categorized in extrinsic and intrinsic factors. Intrinsic factors include balance impairment, neurological disorder, sensory deterioration, postural hypertension and medication use. Extrinsic factors include ill fitted footwear, slippery surfaces, inappropriate furniture and light problems. Balance impairment is an important risk factor for falling.

As the age increases, probability of health problems and functional disability increases due to sedentary life style that might intensify the general physical de conditioning and further reduce their balance. Impaired balance has been correlated with an increased risk of falls and resulting increase in the mortality rate of elderly persons who are prone to falling compared with those who are not prone to falling. Fear of falling is directly related to balance confidence.

There is positive relationship between balance control or motor and sensory system function in order to understand the cause of falling and create effective strategy to prevent fall in the elderly population. Age related changes in postural response to forward slip had shown that balance control was reduced in elderly people as compared with young people. 


\section{The Role of Cognitive Factors in Old Age Balance Impairments}

Sensory system deteriorates with age, increased attention is allocated to "HIGHTEN" the signal coming from this system in order to gain necessary information for postural control. ${ }^{(11,23)}$ Older adults show marked reduction in the ability to perform the postural and cognitive task simultaneously. Greater postural instability when performing dual task has been reported in stance phase and during obstacle avoidance in gait.

In the task of walking, Whit tall found that concurrent task like memorization influenced both step time and step length and these effects are age dependent. Frail elderly people stops walking when they start a conversation with walking companion because walking demands attention and they stops when they are expecting to do two things simultaneously. Maintenance of balance and limb coordination during locomotion requires a complex interaction of sensory input and motor output. Sensory system monitors the location of the whole body Centre of Gravity relative to foot-ground centre of pressure provides orientation in space and monitors the environment. Motor system provides appropriate, coordinated muscular activation and force generation. When brain is engaged the effect is a synchronized movement but when a person is required to perform synchronized movement with divided attention, the performance deteriorates.

Dual tasks methods or paradigms have been used to examine the relative attention demands associated with different types of postural tasks. Changes in the secondary tasks are used to infer task dependent changes in attention demands. So attention demands vary as the function of task complexity, age, and balance abilities. Dual task requires the participants to perform a multiple tasks simultaneously which have been used to investigate the effects of cognitive task on postural control and vice versa. Age related change on dual task is that older subjects have more difficulty in coordination of multiple tasks. This is because of lack of control or attention flexibility. ADLs (Activity of daily living) require maintaining balance during the performance of several concurrent tasks. Dual task training has been used to that allows for the practice of multitask coordination. Inability to perform concurrent task is contributing factor to instability and falling in many older adults who are living in residential care facilities.

A study was conducted in a small town of Haryana on institutionalized older adults who are living in residential care facilities by physiotherapy student in which Dual task training under various sets of instruction that is Fixed priority and Variable priority instruction sets effects the balance.

In Fixed priority instruction set same amount of attention has been given on both tasks i .e on balance tasks and cognitive tasks at all the time. In variable priority instructional set, attention switches between the tasks i.e on the balance tasks and on the cognitive tasks. The efficacy of these two task training strategies is for the acquisition, retention and transfer of task coordination skills in older adults. 
The Role of Cognitive Factors in Old Age Balance Impairments

Tinneti Performance Oriented Mobility Assessment (POMA) was used to for Balance assessment and Balance is defined as the ability of the elderly people to score19 to 24 in the Tinnetti performance oriented mobility assessment (TPOMA).

Mini mental status examination: The MMSE evaluates general cognitive ability, including orientation to date, registration (immediate recall), attention and calculation, recall of 3 words and language, with a score of 24 suggesting decreased cognitive ability (e.g dementia). The MMSE has been shown to have a good test-retest reliability with the same $(r=.887)$ of different $(r=.824)$ examiners.

Experimental hypothesis: There will be a significant improvement in balance of the elderly population following dual task training under variable priority instruction set.

\section{REVIEW OF LITERATURE}

Demos and Wicken (1980) Lintern et al (1991), demonstrated that whole task or dual task training involves practicing both task simultaneously. Dual task training enables the older adults to develop task coordination and attention control. So dual task training is necessary to reduce the dual task decrements that are found when two tasks are performed together. Crack and Byrd (1982), McDown et al (1986) on found that older adults have more difficulty in performing two tasks at once. Assessment of complex motor tasks is sensitive to decline in cognitive function even at an early stage. Whittal et al (1987) on Senile dementia and Alzheimer diseases; an important risk factor for serious falls, found that while walking concurrent cognitive tasks (memorization, singing) influenced both step time and step length and these effects are age dependents. Decline in the muscle activity when performing secondary task suggests that less attention processing capacity was available for balance control during dual task paradigm.

Tinnetti M et al (1990) suggested that secondary factors includes nutrition, exercises, insults and pathologies that effects our mind and body, contributes to ageing. Hawkins et al (1992) and Kortling (1991) et al found that older adults have more difficulty in the management or coordination of multiple tasks. Lack of control or attention flexibility in which older adults had more difficulty than young adults when they required switching rapidly between auditory and visual task. Gopher et al (1992) compared the efficacy of two different dual task training. Variable priority training and fixed priority training, in acquisition of coordination skills, variable priority training led to be better performances on training and transfer task than did fixed priority training. Burlington et al (1998), accepts that sensory system such as visual, vestibular and somato sensory system play important role in postural control and postural balance. As the age increases, the function of sensory system deteriorates.

Sela et al (2001) and Baddley et al (1991) suggested that testing the limits of attention dependent performances in adequate dual task situation can reveals the increases the risk of falling in 


\section{The Role of Cognitive Factors in Old Age Balance Impairments}

cognitively impaired patients. Stelmech and worringhams et al (1999) postulated that effectiveness of integrative mechanism responsible for coordination of vestibular, visual and proprioceptive input that deteriorates with increasing age. Sergio Della Sala et al (2001) revealed that cognitive such as episodic memory and response inhibition are sensitive to the effects of normal ageing. Chirtian Marburgur, Peter et al (2002) found that among those who fall, cognitively impaired older adults carries high risk of morbidity and mortality. Weerdestenyn et al (2003) conducted a study to observe the Effects of Divided attention on Gait stability following concussion. The sample included in the study was suffering from cognitive impairment following traumatic brain injury. The result of the study reported that divided attention decreases obstacle avoidance while walking.

\section{Protocol}

A sample of convenience consisted of 30 older adults included in the study. All Subjects were randomly divided in to two groups 15 subjects each with no age and sex difference. Group 1 was given Dual task training with fixed priority instruction set. Group 2 was given Dual task training with variable priority instruction set consisted of 12 sessions. The subjects were assessed on the basis of "Tinneti performance oriented mobility assessment (TPOMA). Group 1 practiced the set of balance tasks while simultaneously performing secondary tasks. They were directed to maintain attention on both the tasks i .e postural as well as secondary task at all the time. Group 2 received same set of exercises as group 1 but under different instruction set. During each session, half of the training was done with focus on the postural task performance and half had focused on the secondary task performance.

During these sessions in both groups, data on performance accuracy in the secondary task were recorded in terms of numbers of missteps. (In novel task) which was not directly trained.

1. To confirm that patient really allocate attention to one task or not

2. To see the improvement of the performance on this task.

In both groups three days session per week was given. Each session lasting for 45 minutes resulting in 12 sessions balance training program. Balance measurement will be taken before training, second week of training (interim) and then after training of 4 weeks. Similarly novel task performance was taken before exercise and after 4 weeks exercise.

\section{Data Analysis}

Data was analyzed using SPSS 13.0 software packages. The balance performances were examined under fixed and variable priority instruction using paired sample $t$-test within the group and independent sample $\mathrm{t}$ - test between the groups. The $\mathrm{p}-$ value was set at a significance level of $\mathrm{p}<0.05$. 


\section{RESULT AND DISCUSSION}

The result of the study supported the hypothesis that there is significant improvement in balance of institutionalized elderly people who received dual task training with variable priority instruction set. ( $p<0.001$ ). The use of validated measurement tool like Tinnetti performance oriented mobility assessment on balance allowed clearer interpretation of the results. There is improvement in TPOMA Scores after 4 weeks training program in both groups. Dual task training has been used as it suggest that the sensori motor processing essential for motor control that require attention resources.Dysfunction in the sensory and the motor system contributes to increased risk of falling among the age of 65 years or more year of age. Instability and fall rate increased in balance impaired older adults when they performed concurrent task in the environment in which sensory information related to postural control was reduced.

There is more significant improvement in group 2 who received dual task training with variable priority instruction set. Our mean data after four weeks exercise program are on the upper end of the range in TPOMA in group 2 who received variable priority instruction set as compare to group 1 who received fixed priority instruction set.

Balance impairment is a major contributor to falls in older adults over 65 years of age and a growing body of evidence has confirmed the importance of cognitive factors to impaired balance among older adults. There is more significant improvement in group 2 who received dual task training with variable priority instruction set as compare to group 1 who received dual task training under fixed priority instruction set . One possible explanation of this outcome is that task coordination (strategies that people might use to coordinate dual task performance) was included in the balance training under dual task condition.

According to task coordination and management hypothesis, coordinating and managing multiple task is crucial for the dual task performances and this ability might be reduced in elderly people. This dual task balance training is necessary to optimized stability during performance of concurrent tasks Hawkin et al also illustrated that because of lack of control or attention flexibility, older adults had more difficulty in performing dual tasks. Jenning et al found that older adults are less well prepared for rapidly presented stimuli when required to perform two concurrent tasks. So the age related differences in task coordination or attention flexibility is also consistent with finding that older adults exhibit less flexibility than younger adults.

Subjects who received dual task training under variable priority instruction set also showed improvement on a novel task performance (untrained task). Gopher et al supported the hypothesis that improvement in the novel task performance is the result of development of improved dual task processing skills (ability to allocate attention ) and this skills can be generalized to other task dual task that are not directly trained . So this suggests that explicit instruction regarding attention focus should be included when training balance under dual task 
condition with variable priority instruction set. During each session, half of the dual task training was done with attention focus on the postural tasks and half of the training was done with attention focus on the secondary tasks.

Our results supports the fact that dual task training with variable priority instruction set could improve balance performance through specific type of training and improvement of dual task processing skills can be generalized to novel tasks. Variable priority subjects showed faster acquisition of skilled performance on the task and higher level of mastery in acquisition and transfer of dual task skills can be aided by embedding within variable priority instruction set as compared to fixed priority instruction set.

\section{Clinical Relevance}

The clinical significance of this study is that as residents of elderly population residing in institution usually adopts a sedentary life style making them prone to physical de conditioning and decline in motor performance when there is competition for attention resources between to task performed under dual task condition. Balance confidence affects the balance performances. So increasing the balance confidence through balance training exercises can affects the balance performance which has important implication for the development of rehabilitation that aims to improve balances confidence and diminish its impact on the functionality in elderly population .in order to performed multiple task safely, older adults with balance impairment may be

restricted to limited set of environment condition in which sensory condition are optimal. To prevent fall, older adults must be able to maintain postural stability while performing multiple tasks Dual task training with varying instruction set could be referred so as to prevent that further deterioration in balance and risk of falls incidence in institutionalized elderly population.

\section{CONCLUSION}

The study showed significant improvement in balance of the elderly population following dual task training under fixed and variable priority instruction set. But improvement is more marked under variable priority instruction set. Also showed improvement in novel task which are not directly trained. This shows that cognitive factors do play an important role in maintaining balance and coordination. Any impairment related to sensory system or cognition leads to balance impairments and increased risk for falls.

\section{Acknowledgments}

The author appreciates all those who participated in the study and helped to facilitate the research process.

Conflict of Interests: The author declared no conflict of interests. 


\section{REFERENCES}

Albert B et al (1992) Mobility impairment in the elderly challenges for biomechanics research. 25; $519-528$.

Ane Shumway Cook, Marjorie H Woollacott (2006). Training of Balance under single \& dual task condition. In older adults with balance impairment. Journal of Physical Therapy, 86, $269-281$.

Brauer SG, Woollacott M, Shumway-Cook. Interacting effects of cognitive demand and recovery of postural stability in balance impaired elderly people. J Gerontol A Biol Sci Med Sci. 2001 56: M489-M496.

Brown M et al (1991). Effect of low intensity exercise program on selected physical performance characteristics of 60 to 71 years of age. Ageing . 3; 129 -139.

Brown Woollacot MH.(1999 ) Attentional demands and postural recovery. Effect of ageing . $J$ Ger Biol Med Sci. 54:165-171.

Camball, Borrie et al (1990). Circumstances and consequences of falls in independent community-dwelling elder adults, Age-Ageing, 19:136-141.

Cralk et al (1977). Ageing and dual task performance. p 83 - 85 .

Dela sala et al (2001). Theoretical and practical implication of dual task performance in Alzheimer diseases, 8, 1479 -1481.

Ffiateron et al (1990), high intensity strength training program ; effect on skeletal

Ge wu et al (1998). The relation between age related changes in neuro musculoskeletal system and dynamic postural responses to balance disturbance, 53;320 -326 .

Geurts ACH et al. (1991) Dual task assessment of reorganization in persons with lower limb amputation. Arch phys. Med Rehab. 2; 155-161.

Guide to physical therapy practice. 2nd (2001). 81; 731 -744.

Kellong int. work group prev. Falls by elderly, the prevention of falls in the later life. 34, 1-24.

Kerr B, Condon SM, Mc Donald (1985). Cognitive Spatial processing and regulation of posture. J Exp Psychol App. 11: 617-622

Klaus et al (2003) cognitive impairment decreases postural control during dual tasks in geriatric patients with a history of severe falls. J of American Society. $51 ; 1638$-1644

Kramer, larish et al (1991). Training for executive control; task coordination strategies and ageing. 617 -618.

Lundin -olsson et al (1997) Stop walking when talking as a predictor of fall in the elderly people. the lancet, 349; $617-622$.

Marjorie woollacott et al (2002). Attention and the control of posture and gait; a review of an emerging area of research. 16, $1-14$.

Naznin kerns et al (2005). Influence of a cognitive task in postural stability on children with or without attention deficit /hyperactivity disorder; preliminary investigation. 57; 78 - 83.

Nickens H (1982) Intrinsic factors in following among elderly. Archive internal medicine; 145: 1089 - 1093.

P. O. Jantti et al.(1993).Fall among elderly nursing home residents. $J$.of public health .107; 89 96.

Pei - fang tang et al.(1998).Inefficient postural responses to unexpected slips during walking in older adults. J. of Gerontology. 53 A, M471 - M480

Pitts. DG. et al (1982). The effects of aging on selected visual function. Modern Ageing Research; 131 -160. 


\section{The Role of Cognitive Factors in Old Age Balance Impairments}

Rankin et al (2000) cognitive influence on postural stability; A Neuro muscular analysis in young and older adults. 55 (A) M 112 -119.

Richard C. nelson et al (1980). Falls in the elderly, emergency medicine clinics of America. 8; $309-325$.

Richard f et al (1986). Hearing impairment and cognitive decline in senile dementia of the Alzheimer disease. J. American Geriatric society. 34; 207 -210.

Richard w. satin et al (1992). Falls among older persons; a public health perspective. Annu. rev. publ. health. 13, $489-508$.

Roberta $\mathrm{M}$ et al (2003) Effect of cognitive and motor tass on postural stability in parkinsonism diseases; A posturographic study . 18, 652 -658 .

Shumway - Cook et al. (2000). Attentional demands \& postural control. Effects of sensory context. J Gerontol A Biol Science Med Sci; 55 A: M10-M16.

Shumway cook et al (1986) Aging and postural control; changes in sensory organization and muscular coordination. Aging and human development. 23 (2) 97 -112.

Stephaine S. Y. et al .2002 ) Did mobility and balance of residents living in private old age home improve after a mobility exercise program a pilot study Hongkong physiotherapy journal . 20; 15 -19.

Stlimech et al (1989) Age related decline in postural control mechanism. 29 (3) 205 - 223.

Sullivan SB (1999); Assessment of motor function. Chapter 8, edition 4. Physical rehabilitation, $127-212$.

Tideiksaar r. et al (1998). Falls and instability in the elderly. Neurorehabilitation. 3; 51 -61.

Tinnetti ME et al. Multifactor intervention to reduce the risk of falling among elderly people living in the community N. England Journal of Med 1991; 331: 821-827.

Tinnetti ME Risk factor for fall among elderly population. Journal Medicine N England NZ. 1998; 319: $1701-1707$

Tonya M parker et al (2004). The effect of divided attention on gait stability following concussion. $1-6$.

Verghese J. Buschke H Viola et al (2002). Validity of divided attention tasks in predicting falls in older individuals a preliminary study Journal American Geriatric Society, 50:15721576.

Whanger et al (1974) Clinical correlates of the vibratory sense in elderly adults. $J$ Gerontology, 29; $39-45$.

Whitney, S.L. (1998) A view of balance instrument for older adults. The American journal of occupational therapy, 52; $666-671$.

How to cite this article: Yadav P (2017), The Role of Cognitive Factors in Old Age Balance Impairments, International Journal of Indian Psychology, Volume 4, Issue 2, No. 89, ISSN:2348-5396 (e), ISSN:2349-3429 (p), DIP:18.01.093/20170402, ISBN:978-1-365-74162-3 